\title{
Identification of thioridazine, an antipsychotic drug, as an antiglioblastoma and anticancer stem cell agent using public gene expression data
}

\author{
H-W Cheng ${ }^{1}$, Y-H Liang ${ }^{2}$, Y-L Kuo ${ }^{3}$, C-P Chuu ${ }^{4}$, C-Y Lin ${ }^{4,5}$, M-H Lee ${ }^{1}$, ATH Wu ${ }^{6}$, C-T Yeh ${ }^{7}$, El-T Chen ${ }^{2}$, J Whang-Peng ${ }^{8}$, C-L Su ${ }^{*, 9}$ and \\ C-YF Huang, ${ }^{* 1,2}$
}

Glioblastoma (GBM) is a common and malignant tumor with a poor prognosis. Glioblastoma stem cells (GSCs) have been reported to be involved in tumorigenesis, tumor maintenance and therapeutic resistance. Thus, to discover novel candidate therapeutic drugs for anti-GBM and anti-GSCs is an urgent need. We hypothesized that if treatment with a drug could reverse, at least in part, the gene expression signature of GBM and GSCs, this drug may have the potential to inhibit pathways essential in the formation of GBM and thereby treat GBM. Here, we collected 356 GBM gene signatures from public databases and queried the Connectivity Map. We systematically evaluated the in vitro antitumor effects of 79 drugs in GBM cell lines. Of the drugs screened, thioridazine was selected for further characterization because it has potent anti-GBM and anti-GSCs properties. When investigating the mechanisms underlying the cytocidal effects of thioridazine, we found that thioridazine induces autophagy in GBM cell lines, and upregulates AMPK activity. Moreover, LC3-II was upregulated in U87MG sphere cells treated with thioridazine. In addition, thioridazine suppressed GBM tumorigenesis and induced autophagy in vivo. We not only repurposed the antipsychotic drug thioridazine as a potent anti-GBM and anti-GSCs agent, but also provided a new strategy to search for drugs with anticancer and anticancer stem cell properties.

Cell Death and Disease (2015) 6, e1753; doi:10.1038/cddis.2015.77; published online 7 May 2015

Glioblastomas (GBM), the most common and most aggressive primary brain tumors in adults, are classified as grade IV astrocytomas by the World Health Organization and account for $54 \%$ of all gliomas. ${ }^{1}$ Surgery is typically followed by radiation therapy and chemotherapy with temozolomide (TMZ), which has been in clinical use since $2005 .^{2,3}$ Despite this multimodal approach, the median survival time of GBM patients is $\sim 14.6$ months. ${ }^{3}$ Therefore, a large number of new drugs are in development for GBM treatment.

Instead of focusing on a single drug target, using a batch of genes to query the Connectivity Map (Cmap, http://www. broad.mit.edu/cmap/) may not only allow multiple targets to be considered simultaneously, but it may also identify potential new drugs. Cmap is a database that provides $\sim 7000$ microarray expression profiles (conducted on Affymetrix
HG-U133A arrays) from four different cancer cell lines treated with 1309 molecular drugs. Of the 1309 drugs included in Cmap, most are currently used in clinical treatment or are welldeveloped drugs; thus, we can rapidly identify potential drugs and proceed to clinical trial.

Thioridazine is an antipsychotic drug and is widely used to treat schizophrenia and psychosis. Recently, it has been shown that patients with schizophrenia have a lower risk of getting cancer $(1.93 \%)$ than patients without schizophrenia $(2.97 \%) .{ }^{4}$ In addition, inverse cancer comorbidity has been reported in people with certain CNS disorders, and pharmacological treatments is one of possible explanations. ${ }^{5}$

Using in silico drug screening via Cmap followed by empirical validations, we discovered that thioridazine can reduce the viability of GBM cells and GBM stem cells, induce

\footnotetext{
${ }^{1}$ Institute of Biopharmaceutical Sciences, National Yang-Ming University, Taipei, Taiwan; ${ }^{2}$ Department of Biotechnology and Laboratory Science in Medicine, National YangMing University, Taipei, Taiwan; ${ }^{3}$ Department of Computer Science and Information Engineering, National Taiwan University, Taipei, Taiwan; ${ }^{4}$ Institute of Cellular and System Medicine, National Health Research Institutes, Miaoli, Taiwan; ${ }^{5}$ Institute of Cancer Research, National Health Research Institutes, Miaoli, Taiwan; ${ }^{6}$ Department of Radiation, School of Medicine, Taipei Medical University, Taipei, Taiwan; ${ }^{7}$ Graduate Institute of Clinical Medicine, Taipei Medical University, Taipei, Taiwan; ${ }^{8}$ Municipal Wan Fang Hospital, Taipei Medical University, Taipei, Taiwan and ${ }^{9}$ Program of Nutritional Science and Education, Department of Human Development and Family Studies, National Taiwan Normal University, Taipei, Taiwan

${ }^{*}$ Corresponding author: C-L Su or C-YF Huang, Program of Nutritional Science and Education, Department of Human Development and Family Studies, National Taiwan Normal University, No. 162, Section 1, He-ping East Road, Taipei 10610, Taiwan. Tel: +886 277341436 or +886 22826 7904; Fax: +8862 23639635 or +886 228224045 ; E-mails: chunlisu@ntnu.edu.tw or cyhuang5@ym.edu.tw

Abbreviations: AMPK, AMP-activated protein kinase; AVOs, acidic vesicular organelles; BCRC, Bioresource Collection and Research Centre; Bip, binding immunoglobulin protein; c-Caspase-3, cleaved-Caspase-3; CHOP, C/EBP homologous protein; Cmap, connectivity map; c-PARP, cleaved-Poly (ADP-ribose) polymerase; DAPI, 4',6-diamidino-2-phenylindole; DMEM, Dulbecco's modified Eagle's medium; DMSO, dimethyl sulfoxide; DRD1, dopamine receptor D1; DRD2, dopamine receptor D2; EGF, epidermal growth factor; GBM, glioblastoma; GEO, Gene Expression Omnibus; GFP, green fluorescent protein; GSCs, glioblastoma stem cells; HBSS, Hank's balanced salt solution; HRP, horseradish peroxidase; IRE1 $\alpha$, inositol-requiring enzyme 1 alpha; JNK, c-Jun N-terminal kinases; LAMP-2, lysosome-associated membrane protein 2; LC3, microtubule-associated protein 1 light chain 3 (MAP1LC3); MGMT, $0^{6}$-methylguanine-DNA methyltransferase; mTOR, mammalian target of rapamycin; MTT, 3-(4,5-dimethylthiazol-2-yl)-2,5-diphenyltetrazolium bromide; NCBI, National Center for Biotechnology Information; p70S6K, p70 ribosomal protein S6 kinase; PBS, phosphate-buffered saline; PI3K, phosphatidylinositol 3-kinase; Raptor, regulatory associated protein of MTOR; SAPK, stress-activated kinases; SDS, sodium dodecyl sulfate; TCTP, translationally controlled tumor protein; TMZ, temozolomide; VEGF, vascular endothelial growth factor

Received 14.11.14; revised 15.2.15; accepted 17.2.15; Edited by A Stephanou
} 
autophagy and affect the expressions of related proteins in GBM cells. Thus, thioridazine has potential to treat GBM.

\section{Results}

Using GBM gene signatures to identify drugs for GBM via Cmap. We hypothesized that if a drug treatment could at least partially reverse the gene expression signature of GBM, it might have the potential to inhibit pathways essential in the formation of GBM and could therefore be used to treat GBM. We combined data from five publicly available microarray data sets from the National Center for Biotechnology Information (NCBI) Gene Expression Omnibus (GEO). All five data sets were published previously. ${ }^{6-10}$ The data sources are summarized in Table 1 and data analysis is described in the Materials and Methods section. Briefly, differentially expressed genes appear in all five data sets, including two upregulated genes (COL4A1 and MTHRD2) and eight downregulated genes (KIAA1598, CHN2, EPB41L3, GNAI1, GRM3, SH3GL3, RAPGEF5 and $R A B 40 B$ ). The total list of differentially expressed genes is found in Supplementary Information, Supplementary Table S4. We then used the GBM gene signatures (determined by comparing the GBM gene expression pattern to the normal brain gene expression pattern) from each data set to query the Cmap (with negative enrichment scores and $P<0.05$ ) as described in the Materials and Methods section. Figure 1a shows the 215 drugs identified from the fusion of all five data sets, and the list of these drugs is found in Supplementary Information, Supplementary Table S1.

\section{Characterization of potential drugs for GBM via cell} viability and clonogenic assays. To examine whether the 215 predicted drugs have antitumor effects on malignant gliomas, we randomly tested 65 potential drugs in the GBM8401 cells and determined the cell viability by an 3-(4,5-dimethylthiazol-2-yl)-2,5-diphenyltetrazolium bromide (MTT) assay. If the $\mathrm{IC}_{50}$ value of the drug was less than
$10 \mu \mathrm{M}$, we defined the drug as an 'effective' drug. For example, Supplementary Information, Supplementary Figure S2 illustrates the MTT results from cells treated with thiostrepton, thioguanosine, parthenolide and bepridil; the $\mathrm{IC}_{50}$ value for thiostrepton is $0.8 \mu \mathrm{M}$. The 'ineffective' drugs $\left(\mathrm{IC}_{50}\right.$ value $\left.>10 \mu \mathrm{M}\right)$ were tested further using clonogenic assays. Effectiveness in the clonogenic assay was defined as a reduction in the number of colonies by more than $50 \%$ at $10 \mu \mathrm{M}$. The quantifications of the clonogenic assays for four antipsychotic drugs, including prochlorperazine, chlorpromazine, thioridazine and trifluoperazine, are illustrated in Figure 1b. We also tested an additional 14 antipsychotic drugs (Figure 1c); in total, 79 drugs were tested. Figure 1c summarizes the results of 34 effective drugs. We classified these effective drugs (25 effective drugs in the MTT assay and 9 effective drugs in the clonogenic assay) into six different functional groups (anti-inflammation, antibiotics, antipsychotics, cardiovascular drugs, chemotherapeutic drugs and others), as summarized in Table 2.

Analysis of cell viability in GBM cell lines after treatment with antipsychotic drugs. We chose the antipsychotic drugs as our top priority for the following three reasons: (1) antipsychotic drugs can across the blood-brain barrier; (2) one of the studies collected through data mining of the National Health Insurance Research Database in Taiwan noted that patients with schizophrenia have a lower risk of developing cancer. ${ }^{4}$ Thus, we speculated that there might be some correlation between cancer and antipsychotic drugs; and (3) antipsychotic drugs could reverse, at least in part, the gene expression signature of cancer stem-like cells as observed in the Cmap analysis (Supplementary Information, Supplementary Table S5). These arrays of cancer stem-like cells were from three published studies. ${ }^{11-13}$ Therefore, to systematically examine the cytotoxicity of antipsychotic drugs on malignant glioma, we expanded the collection of the seven antipsychotic drugs predicted by Cmap (acepromazine, amoxapine, chlorpromazine, fluspirilene, prochlorperazine, thioridazine and trifluoperazine) to

Table 1 Summary of five cohorts of GBM data sets used for Cmap analysis

\begin{tabular}{|c|c|c|c|c|c|}
\hline GEO no. & GSE4290 & GSE7696 & GSE14805 & GSE15824 & GSE16011 \\
\hline Pubmed ID & 16616334 & 18565887 & 19139420 & 21406405 & 19920198 \\
\hline Journal & Cancer Cell & Clin Oncol & Neuro Oncol & Cancer Res & Cancer Res \\
\hline Up probe sets no. & 120 & 76 & 364 & 67 & 462 \\
\hline Down probe sets no. & 286 & 194 & 506 & 44 & 538 \\
\hline Brain tumor (GBM) & 81 & 70 & 34 & 12 & 159 \\
\hline Normal brain & 23 & 4 & 4 & 2 & 8 \\
\hline Array platform & HG-U133_Plus_2 & HG-U133_Plus_2 & HT_HG-U133A & HG-U133_Plus_2 & HG-U133_Plus_2 \\
\hline
\end{tabular}

The microarray data were downloaded from the GEO. The differentially expressed gene signatures from each data set were used to query the Cmap

Figure 1 Integrated bioinformatics and biochemical analyses reveal potential drugs for GBM. (a) This Venn diagram represents the number of drugs found using the five GBM data sets used to query the Cmap database. (b) GBM8401 cells were treated with prochlorperazine, chlorpromazine, thioridazine and trifluoperazine at concentrations ranging from 1 to $10 \mu \mathrm{M}$, and cell viability was determined using a clonogenic assay. (c) Summary of drugs tested on GBM8401 cells. Of the 215 potential GBM drugs as predicted by Cmap, we randomly selected 65 potential drugs and 14 antipsychotic drugs based on the availability of well-known scientific names and manufacturers, and we tested the cytotoxicity of these drugs in two GBM cell lines via an MTT and/or a clonogenic assay. There were 25 effective drugs (IC $50<10 \mu \mathrm{M})$ in the MTT assay and 9 drugs effective $\left(\mathrm{IC}_{50}<10 \mu \mathrm{M}\right)$ in the clonogenic assay. (d) GBM8401 cells were treated with $10 \mu \mathrm{M}$ various antipsychotic drugs for $72 \mathrm{~h}$. Cell viability was determined by MTT assay. (e) GBM8401 and U87MG cells were treated with thioridazine, fluphenazine or temozolomide (the only FDA-approved drug for GBM) at concentrations of 1,5 or $10 \mu \mathrm{M}$ for $72 \mathrm{~h}$, respectively. Cell viability was determined via MTT assay 
a

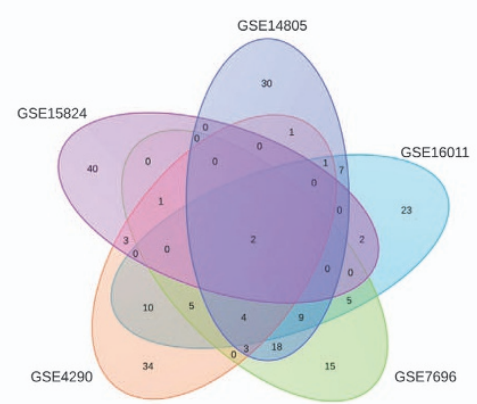

b

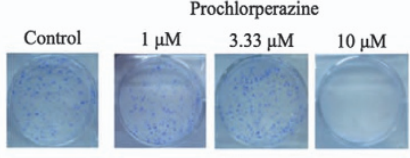

Thioridazine

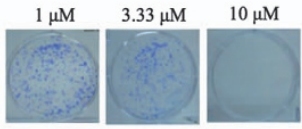

GBM8401-Clonogenic assay

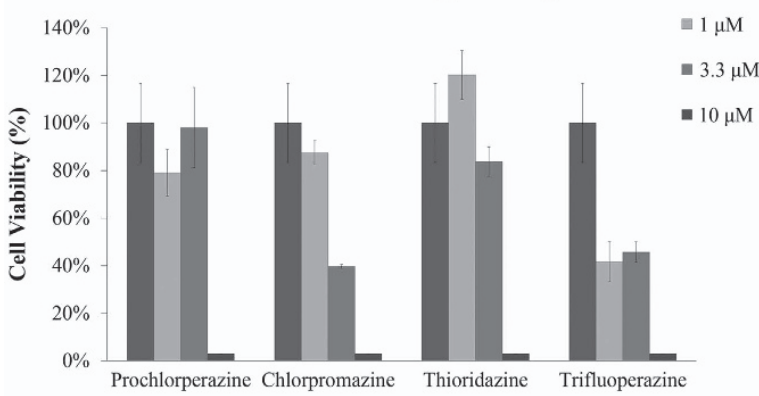

C

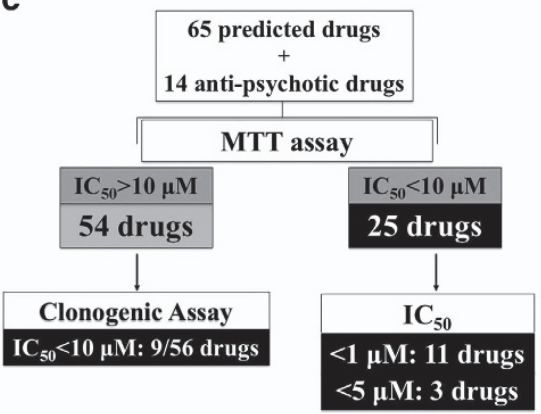

d

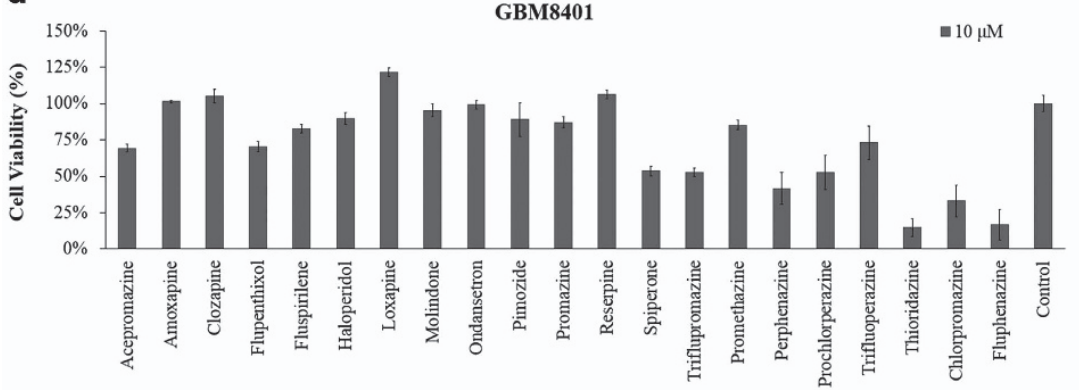

e
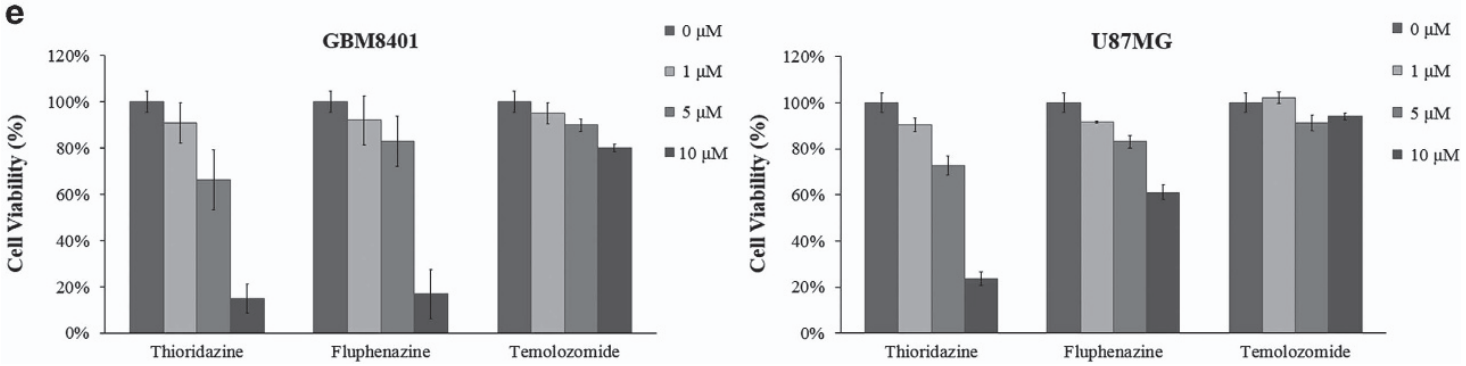
Table 2 Thirty-four effective drugs were classified into six different functional groups

\begin{tabular}{|c|c|c|c|c|c|}
\hline \multirow[t]{2}{*}{ Function } & \multirow[t]{2}{*}{ Drug name } & \multicolumn{2}{|c|}{ PubMed search } & \multicolumn{2}{|c|}{ Experiment } \\
\hline & & Brain cancer & Other cancers & MTT IC ${ }_{50}(\mu \mathrm{M})$ & Clonogenic $\mathrm{IC}_{50}(\mu \mathrm{M})$ \\
\hline \multirow[t]{4}{*}{ Anti-inflammation } & Tanespimycin & V & V & $<0.1$ & ND \\
\hline & 15-delta prostaglandin J2 & V & V & $>10$ & $<10$ \\
\hline & Luteolin & V & V & $<10$ & $<10$ \\
\hline & Parthenolide & V & V & $5 \sim 10$ & $<10$ \\
\hline \multirow[t]{4}{*}{ Antibiotics } & Thiostrepton & V & V & $<1$ & ND \\
\hline & Antimycin A & V & V & $<1$ & $<10$ \\
\hline & Sulconazole & N/A & N/A & $>10$ & $<10$ \\
\hline & Cycloserine & V & V & $<10$ & ND \\
\hline \multirow[t]{6}{*}{ Antipsychotics } & Chlorpromazine & V & V & $5 \sim 10$ & $<3.3$ \\
\hline & Trifluoperazine & V & V & $>10$ & $<1$ \\
\hline & Thioridazine & V & V & $5 \sim 10$ & $<10$ \\
\hline & Prochlorperazine & V & V & $>10$ & $<10$ \\
\hline & Fluphenazine & V & V & $5 \sim 10$ & ND \\
\hline & Perphenazine & V & V & $5 \sim 10$ & $<10$ \\
\hline \multirow{4}{*}{ Cardiovascular drugs } & Piperlongumine & V & V & $<1$ & ND \\
\hline & Propafenone & N/A & V & $>10$ & $<10$ \\
\hline & Phenoxybenzamine & V & V & $>10$ & $<10$ \\
\hline & Amiodarone & V & V & $>5$ & ND \\
\hline \multirow{6}{*}{ Chemotherapeutic drugs } & Daunorubicin & V & V & $<1$ & ND \\
\hline & Camptothecin & V & V & $<0.1$ & $<10$ \\
\hline & Thioguanosine & V & V & $<5$ & ND \\
\hline & 8-azaguanine & V & V & $<10$ & ND \\
\hline & GW-8510 & N/A & V & $<3$ & ND \\
\hline & Ellipticine & V & V & $<1$ & ND \\
\hline \multirow[t]{10}{*}{ Others } & Cloperastine & N/A & $\mathrm{N} / \mathrm{A}$ & $>10$ & $<10$ \\
\hline & Nitrarine dihydrochloride & N/A & $\mathrm{N} / \mathrm{A}$ & $>10$ & $<10$ \\
\hline & Emetine & V & V & $<0.1$ & ND \\
\hline & Tyloxapol & N/A & V & $5 \sim 10$ & ND \\
\hline & Norcyclobenzaprine & N/A & $\mathrm{N} / \mathrm{A}$ & $>10$ & $<10$ \\
\hline & Trichostatin A & V & V & $<0.33$ & ND \\
\hline & Pyrvinium & N/A & V & $<0.1$ & ND \\
\hline & Bepridil & V & V & $5 \sim 10$ & ND \\
\hline & Verteporfin & N/A & V & $<3.3$ & ND \\
\hline & Vorinostat & V & V & $<1$ & ND \\
\hline
\end{tabular}

Abbreviations: 'N/A', no evidence linked to cancer in PubMed; 'ND', not determined

21 antipsychotic drugs that had data in the Cmap collections. As shown in Figures 1d and 4 drugs (perphenazine, thioridazine, chlorpromazine and fluphenazine) have an $\mathrm{IC}_{50}$ value $<10 \mu \mathrm{M}$. Because perphenazine and fluphenazine were also effective against GBM cells, we also included these two drugs in Table 2. In particular, thioridazine and fluphenazine resulted in more cytotoxicity. To confirm the effect of the drugs in malignant glioma cell lines, the GBM8401 and U87MG cells were treated with thioridazine and fluphenazine at concentrations ranging from 1 to $10 \mu \mathrm{M}$ for $72 \mathrm{~h}$, and a dose-dependent decrease in cell growth was observed (Figure 1e). Currently, vascular endothelial growth factor (VEGF) signaling, the epidermal growth factor (EGF) family, the SRC family, platelet-derived growth factor receptorand integrins are being investigated as potential molecular therapeutic targets for GBM treatment. ${ }^{14-16}$ As increase in phosphoEGFR was observed in fluphenazine-treated GBM8401 cells (Supplementary Information, Supplementary Figure S4), thioridazine instead of fluphenazine was used for the following experiments.

Effects of thioridazine on cell autophagy in GBM cell lines. To investigate the mechanisms underlying the cytocidal effects of thioridazine, GBM8401 cells were treated with thioridazine at concentrations ranging from 5 to $15 \mu \mathrm{M}$ or with fluphenazine at concentrations of 10 and $20 \mu \mathrm{M}$ for $24 \mathrm{~h}$.
Following this incubation, we examined protein levels using a microwestern technique, which allows for a quantitative, sensitive and high-throughput (96 different antibodies) assessment of protein levels. ${ }^{17}$ We randomly selected 96 proteins from various pathways. These proteins are primarily involved in the autophagic and cell growth-related pathways (Supplementary Information, Supplementary Table S3). For example, phospho-AMPK (Thr-172) was 3.4-fold upregulated, phospho-mTOR (mammalian target of rapamycin) was 0.58-fold downregulated and phospho-p70S6K (p70 ribosomal protein S6 kinase; Thr-389) was 0.46-fold downregulated. Additional results are summarized in Supplementary Information, Supplementary Table S6. These microwestern results were further validated using the western blot analysis.

It has been shown that GBM cells response better to agents that induce autophagy than apoptosis. ${ }^{18-20}$ To investigate whether the mechanisms of thioridazine undergo autophagy, apoptosis or both pathways in GBM cells, we used western blot analysis to examine the level of LC3-II (a marker of auto phagy), cleaved-Caspase-3 (c-Caspase-3) and cleaved-PARP (c-PARP; markers of apoptosis). The data showed that LC3-II was significantly upregulated in both cell lines treated with thioridazine in a dose-dependent manner, and c-Caspase-3 and c-PARP were also increased at the highest concentration 
(Figure 2a). It should be noted that thioridazine induces autophagy at 5 and $7.5 \mu \mathrm{M}$ in a dose-dependent manner, but does not affect apoptosis at these conditions. The data showed that thioridazine has cytotoxic effect at $7.5 \mu \mathrm{M}$ in GBM cells (Figure 2b). As shown in Figure 2c, thioridazine induced autophagy at $7.5 \mu \mathrm{M}$ in a time-dependent manner, yet apoptosis was not observed at this condition (data not shown). To verify this observation, flow cytometry was used to quantify acid vesicular organelles (AVOs) after thioridazinetreated GBM cells were stained with acridine orange $(A O)$. It revealed that thioridazine induced AVOs accumulation in a dose-dependent manner (Figure 2d). These results further confirm that thioridazine induces autophagy in GBM cells.

To examine whether thioridazine regulates autophagy flux in GBM cells, immunofluorescence assay was used to analyze the colocalization of LAMP-2 (lysosome-associated membrane protein 2; marker of late endosomes/lysosomes) and LC3 in GBM cells after a 24-h thioridazine treatment. The data showed that thioridazine induced LC3-green punctates (Figure 2e). Moreover, extensive colocalization of LC3-green punctates with LAMP-2 was observed in thioridazine-treated GBM cells. These results support that thioridazine induces autophagy flux in GBM cells. Taken together, these results suggest that autophagy has a critical role in thioridazineinduced cytotoxicity of GBM cells.

It is noteworthy that cleavage of caspase-8 and Beclin1 was observed (Figure 2f) and the activity of caspase-8 was upregulated in GBM8401 and U87MG cells treated with higher concentrations of thioridazine (Figure 2g). AMPKinduced phosphorylation and cleavage of Beclin1 have been reported to cause cleavage of caspase 8 , leading to shift autophagy to apoptosis. ${ }^{21}$ These results suggest a possible link between thioridazine-induced autophagy and apoptosis.

Pathway analysis of thioridazine in GBM cell lines. We examined the phosphatidylinositol 3-kinase (PI3K)/Akt/ mTOR pathway, which has known roles in the regulation of autophagy. Interestingly, the data showed that thioridazine affected the PI3K/Akt pathway at $15 \mu \mathrm{M}$ and PI3K, phosphoAkt (Ser-473) and phospho-p70S6K (Ser-424) were down regulated. However, phospho-AMPK (Thr-172) was upregulated in a dose-dependent manner (Figure 3a). These results suggest that the major pathway of thioridazineinduced autophagy may be through AMPK activation in GBM cells. The microwestern (Supplementary Information, Supplementary Table S6) and western (Supplementary Information, Supplementary Figure S3) results indicate that phospho-regulatory associated protein of MTOR (Raptor) was downregulated. Recently, phosphorylation of Raptor, a component of the mTOR complex 1, by AMPK has been reported. $^{22}$ Although the expression of $p-m T O R$ was not significantly different (Figure 3a), thioridazine-induced autophagy may be also through AMPK-induced suppression of Raptor. It is noteworthy that total AMPK was downregulated, although phospho-AMPK (Thr-172) was upregulated (Figure 3a; Supplementary Information, Supplementary Table S6). Recently, it has been reported that thioridazine decreased protein expression of translationally controlled tumor protein (TCTP), ${ }^{23}$ and depletion of TCTP led to phosphorylation of AMPK at Thr $-172 .{ }^{24} \mathrm{Mmi1}$, the yeast homolog of mammalian TCTP, acted as a proteasome inhibitor, protecting protein substrates from proteasomal degradation. ${ }^{25}$ Another possibility may be due to the regulation of AMPK by the ubiquitin proteasome system, in which activating AMPK using AMPK agonists increases expression of ubiquitin ligases (MuRF1 and MAFBx/Atrogin1), and enhances ubiquitination of AMPK. ${ }^{26}$

Recent report demonstrates that ER stress might be an upstream link for autophagy- and apoptosis-mediated cell death in gliomas. ${ }^{27}$ As shown in Figure $3 b$, unfolded protein response activation (accumulation of inositol-requiring enzyme 1 alpha (IRE1a)) and accumulation of ER stressassociated proteins (binding immunoglobulin protein (Bip) and $\mathrm{C} / \mathrm{EBP}$ homologous protein (CHOP)) were observed in thioridazine-treated GBM cells. These results suggest that thioridazine induces ER stress and autophagy.

Effects of thioridazine on DRD2 activity in GBM cell lines. Thioridazine is known to target dopamine receptors in patients with schizophrenia and psychosis. ${ }^{28,29}$ We sought to investigate whether different phenothiazine derivatives (thioridazine, trifluoperazine, prochlorperazine and fluphenazine) might affect dopamine receptor D2 (DRD2) expression in GBM cells. The data showed that both thioridazine and trifluoperazine downregulated the expression of DRD2 in GBM cells, whereas prochlorperazine and fluphenazine had no effect on DRD2. These results suggest that different phenothiazine derivates have different effects on DRD2 in GBM cells. We further identified whether DRD2 expression was associated with autophagy. The data showed that LC3-II was upregulated in GBM cells treated with thioridazine, prochlorperazine and fluphenazine, but not trifluoperazine (Figure 3c). These results suggest that the autophagy pathway induced by these antipsychotic drugs is independent of DRD2 expression in GBM.

Analysis of cell viability in GBM cancer stem-like cells after treatment with anti-psychotic drugs. Our bioinformatics analysis indicated that anti-psychotic drug signatures have a negative (or reverse) correlation with cancer stem-like cell signatures ${ }^{11-13}$ as shown in Supplementary Information, Supplementary Table S5. We first used a flow cytometrybased side population technique to isolate the cancer stemlike cells from the GBM8401 and U87MG cells to examine the potential anti-cancer stem-like cells effects in response to these anti-psychotics drugs. Treatment with thioridazine reduced the percentages ( $>50 \%$ ) of the side population cells in both the GBM8401 and U87MG cells. However, fluphenazine only reduced the percentage of the side population cells in the U87MG cells but not in the GBM8401 cells (Figures $4 a$ and b). In short, we observed that thioridazine was more effective than fluphenazine against the GBM stem-like cells based on the side population assay. To examine whether GBM sphere cells have cancer stem cell properties, TaqMan real-time PCR was used to detect the gene expression of glioblastoma stem cell (GSC) markers in GBM sphere cells. The expression of the GSCs markers, including CD133, CD44 and Nestin, were enhanced in GBM sphere cells (Figure 4c). These results indicate that GBM sphere cells have cancer stem cell properties. 
To investigate whether thioridazine or fluphenazine possess anticancer stem-like cell properties, U87MG cells were cultured in HEScGRO serum-free medium for the duration of the tumor spheroid assay. Sphere cells (cancer stem-like cells) were treated with thioridazine (Figure $4 d$ ) or fluphenazine
(Figure $4 \mathrm{e}$ ) at 10 and $20 \mu \mathrm{M}$ for $24 \mathrm{~h}$. The cell viability was significantly reduced. As described previously, we found that thioridazine induces autophagy in GBM cells; thus, we further investigated whether thioridazine induces autophagy in GBM sphere cells. After a 24-h treatment with thioridazine, LC3-II a
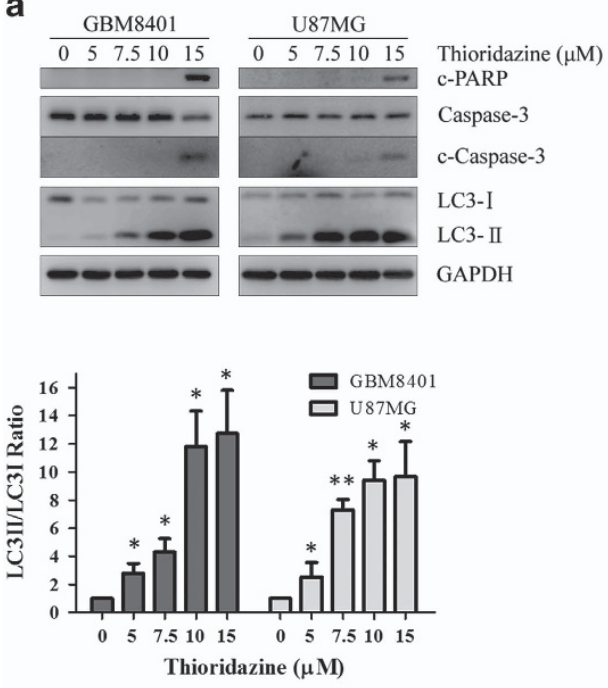

b

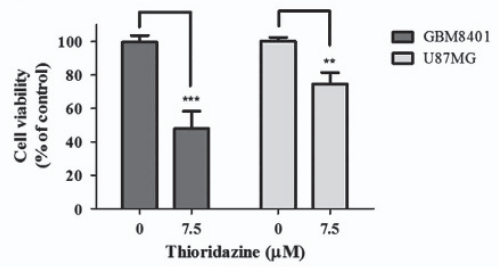

C
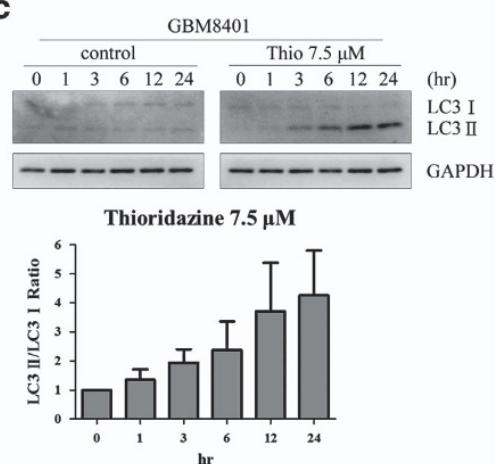

d

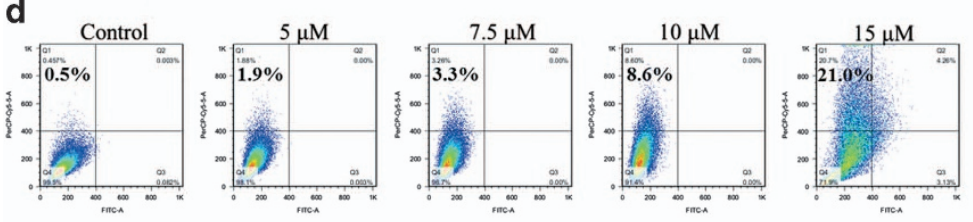

Control

Thioridazine

$\mathrm{THD}+\mathrm{BAF}$
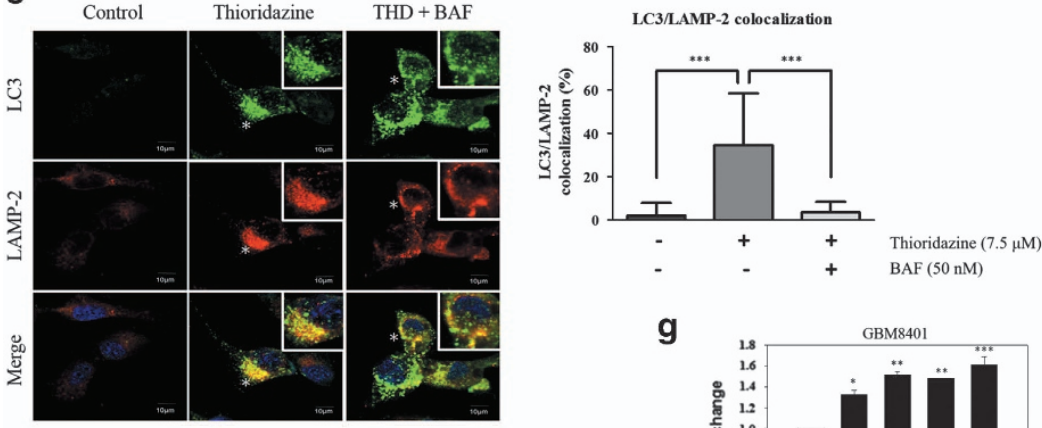

f
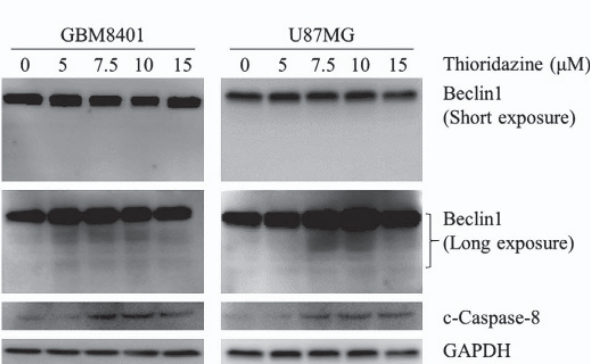

g
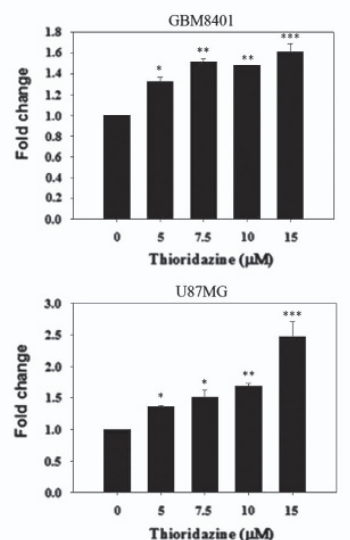
was significantly upregulated in GBM sphere cells in a dosedependent manner (Figure 4f). These results suggest that thioridazine induces autophagy in GBM sphere cells.

\section{In vivo examination of anti-GBM effect of thioridazine.}

To detect the effect of thioridazine in vivo, we tested tumor growth in xenograft mice model. As shown in Figures $5 a$ and b, the tumor size was significantly smaller than those in the control group. To further study the relationship of autophagy in GBM, tumor sections from different groups of mice were examined. A very high expression of LC3-II was observed in thioridazine-treated mice as compared with control mice using immunohistochemistry (Figure $5 c$ ). Overall, we provide strong evidence of thioridazine with autophagy in anti-GBM effect.

\section{Discussion}

Using the Cmap, a powerful bioinformatics tool in drug discovery, we showed that thioridazine, an antipsychotic drug used to treat schizophrenia and psychosis, is an anti-GBM agent. Treatment with thioridazine decreases the viability of GBM cells and cancer stem-like cells. In addition, thioridazine induces autophagy and induces apoptosis at the high concentration. These phenomena may function through G protein-coupled receptors.

TMZ methylates DNA at the N-7 or O-6 positions of guanine residues, damaging the DNA and leading to death in tumor cells. The methylation of the $0^{6}$-methylguanine-DNA methyltransferase (MGMT) gene promoter has been used to predict tumor response to TMZ. ${ }^{30}$ MGMT is a DNA-repair protein that prevents the chemotherapy-induced DNA damage by maintaining the structural integrity of an $\mathrm{O}^{6}$-alkylated base. However, about half of all GBM patients obtained an unmethylated MGMT promoter and therefore respond poorly to $\mathrm{TMZ}$. $^{14,30}$

In this study, we set up three bioinformatics analyses to guide our drug-repurposing pipeline. First, many genomic approaches were used to annotate the disease-causing genes in GBM. ${ }^{31,32}$ Here, we analyzed the GBM gene signature from five microarray studies ${ }^{6-10}$ and used the selected signatures to query the Cmap database. We did not focus on those intersection drugs (Supplementary Information, Supplementary Table S1), for example, 1,4-chrysenequinone and 0173570-0000, because these drugs have not been tested on human subjects. These compounds may take a long time to develop into usable drugs for the treatment of GBM. Second, cancer stem cells are thought to have a role in GBM resistance and recurrence. ${ }^{33}$ Cmap analysis predicted thioridazine to inhibit these GBM cancer stem-like cells (Supplementary Information, Supplementary Table S5). Finally, we took advantage of the multitude of literature available and mined data from the National Health Insurance Research Database in Taiwan and set up our second criteria for drug prioritization. In this study, we noticed that patients with schizophrenia ( 59 000) have a lower risk of developing cancer than the control patients $(\sim 178000) .{ }^{4}$ On the basis of these data integrations and biochemical analyses, we subsequently demonstrated that thioridazine was effective in decreasing the growth of GBM cells and GBM stem-like cells. Moreover, a study reported that thioridazine selectively targets human somatic cancer stem cells capable of leukemic disease initiation, whereas it had no effect on normal blood stem cells. ${ }^{34}$ This further supports our hypothesis that thioridazine is an anti-GBM agent that targets GBM cancer stem-like cells.

Thioridazine has been shown to induce DNA fragmentation and increase Caspase-3 activity; however, higher doses $(6 \sim 50 \mu \mathrm{M})$ are needed to elicit these results in glioma cells. ${ }^{35}$ Here, we clearly demonstrate that thioridazine induces both autophagy and apoptosis in GBM cells. Our data, however, point that autophagy might be a major mechanism underlying the effects of thioridazine. As in GBM sphere cells, treatment of thioridazine also induces autophagy, but not apoptosis. Further, autophagy occurred earlier than apoptosis in GBM cells. In addition, thioridazine appears to inhibit GBM tumor growth effectively and induces autophagy in vivo, suggesting that thioridazine induced autophagy is cytocidal role in GBM.

Using Cmap as an alternative tool for drug discovery could lead to FDA approval of old drugs to treat other disorders. In addition, 443 drugs, which include thioridazine, in the Cmap database have been tested in humans according to the National Health Insurance Research Database in Taiwan. This supports our drug discovery pipeline in drug repurposing to quickly survey survival data and side effect of patients before conducting clinical trials looking for new indications.

\section{Conclusions}

We not only repurposed the antipsychotic drug thioridazine as a potent anti-GBM and anti-GBM cancer stem-like cells agent,

Figure 2 Thioridazine induces autophagy in GBM cells. (a) GBM8401 and U87MG cells were treated with thioridazine at 5, 7.5, 10 and $15 \mu \mathrm{M}$ for $24 \mathrm{~h}$, respectively. c-PARP, Caspase-3, c-Caspase-3, LC3-I and LC3-II were detected by western blotting. GAPDH was used as an internal control. The intensity of LC3-II band density was normalized to LC3I, showing the conversion rate of LC3-I to LC3-II. Bar graph represents the mean of triplicates \pm S.D. ${ }^{*} P<0.05,{ }^{* *} P<0.01$ compared with the control group. (b) GBM8401 and U87MG cells were treated with $7.5 \mu \mathrm{M}$ thioridazine for $24 \mathrm{~h}$. Bar graph represents the mean of triplicates \pm S.D. ${ }^{* *} P<0.01$, ${ }^{* \star *} P<0.005$ compared with the control group. (c) GBM8401 cells were treated with thioridazine at $7.5 \mu \mathrm{M}$ for 1, 3, 6, 12 and $24 \mathrm{~h}$, respectively. LC3-I and LC3-II were detected by western blotting. GAPDH was used as an internal control. The intensity of LC3-II band density was normalized to LC3I, showing the conversion rate of LC3-I to LC3-II. (d) GBM8401 cells were treated with thioridazine at 5, 7.5, 10 and $15 \mu \mathrm{M}$ for $24 \mathrm{~h}$, respectively. Acridine orange-positive cells were quantified by using flow cytometry. Bar graph represents the mean of triplicates \pm S.D. ${ }^{* *} P<0.01$ compared with the control group. (e) U87MG cells were pretreated with or without $50 \mathrm{nM}$ Bafilomycin A1 for $4 \mathrm{~h}$, and then treated with $7.5 \mu \mathrm{M}$ thioridazine for $24 \mathrm{~h}$. Cells were stained with antibodies against LC3 (green) and LAMP-2 (red). Asterisk indicates the colocalization of LC3 and LAMP-2 (yellow). Inset shows magnified view of colocalizations. All merged images together with DAPI staining of DNA (blue). Colocalization was analyzed by confocal microscopy (x60 magnification). Bar graph represents the mean of duplicates \pm S.D., as quantified in at least 25 cells for each experimental condition. ${ }^{* \star *} P<0.005$. Bars $=10 \mu \mathrm{m}$. BAF, Bafilomycin A1. (f) U87MG and GBM 8401 were treated with thioridazine for $24 \mathrm{~h}$. After treatment, the cleavage of beclin1 and caspase-8 was detected using immunoblotting. The GAPDH was used as an internal control. (g) U87MG and GBM 8401 were treated with thioridazine for $24 \mathrm{~h}$. The activity of caspase-8 was determined using luminescence. Bar graph represents the mean of triplicates \pm S.D. ${ }^{*} P<0.05$,

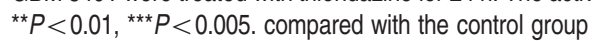


a

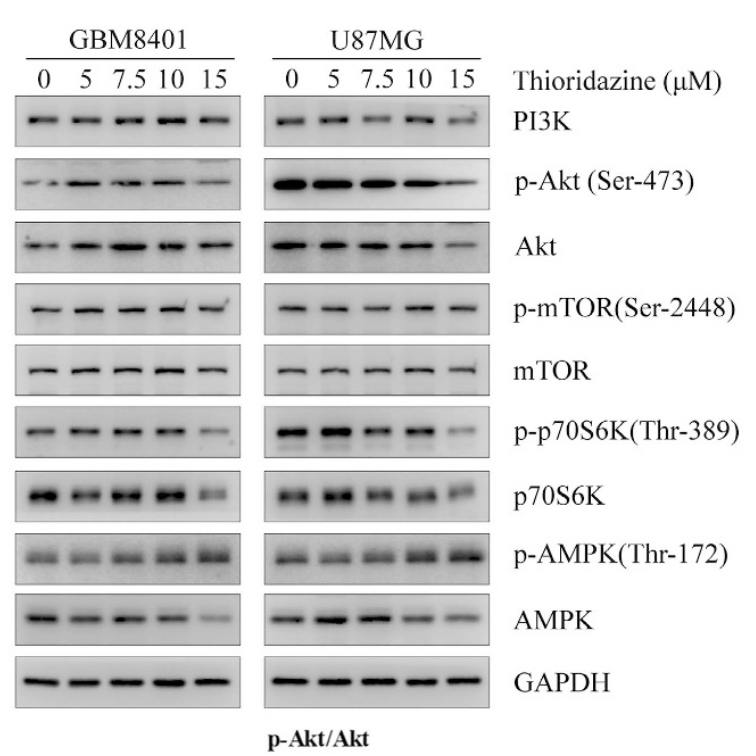

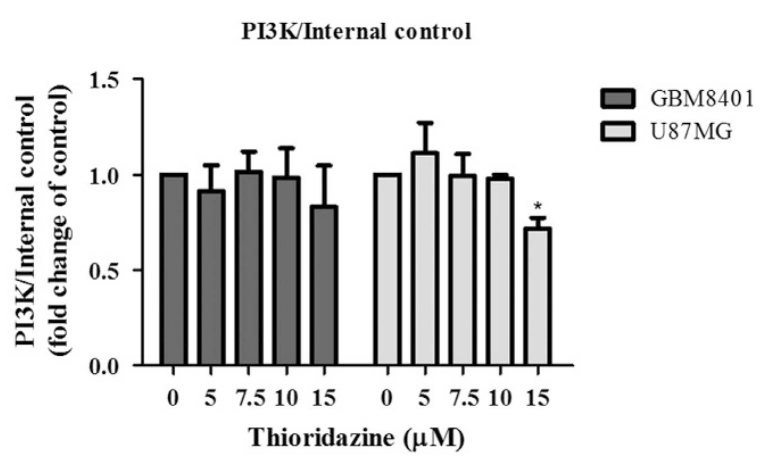

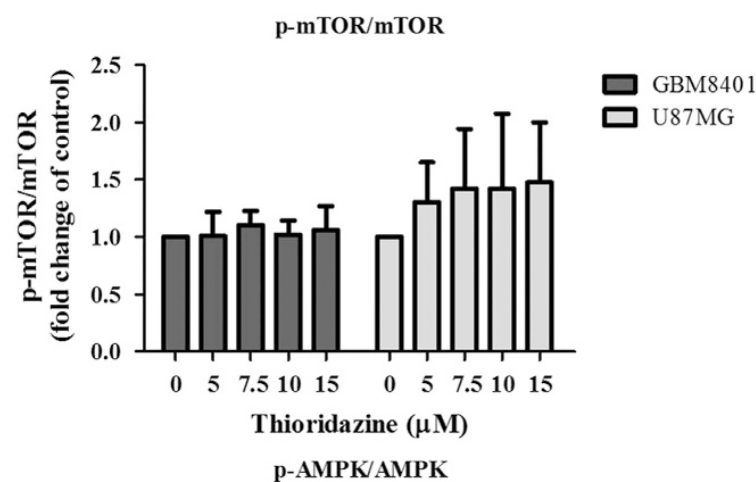

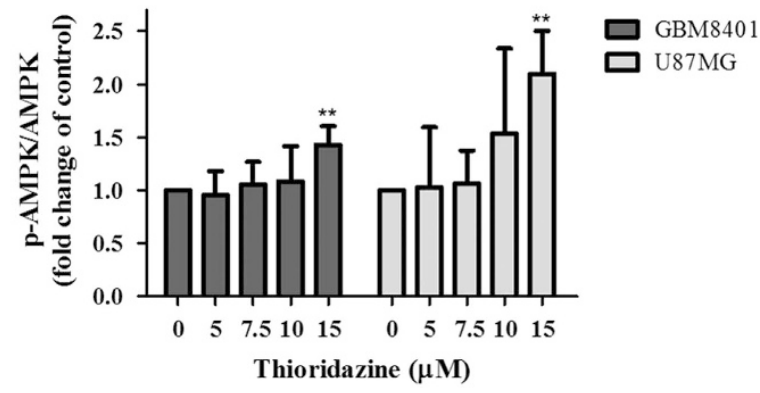

C

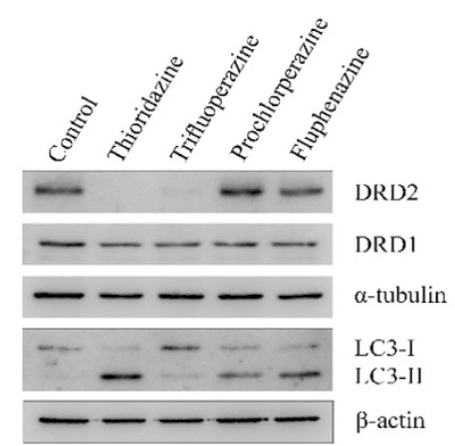

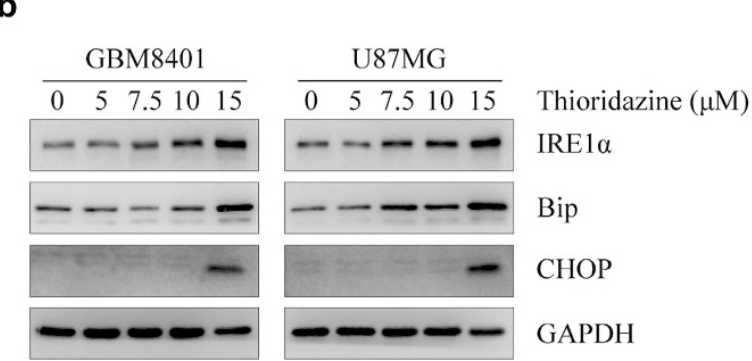


a

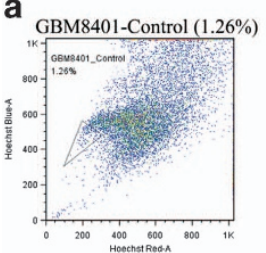

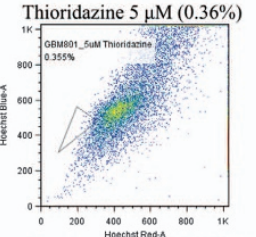

Fluphenazine $5 \mu \mathrm{M}(1.27 \%)$

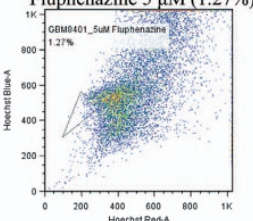

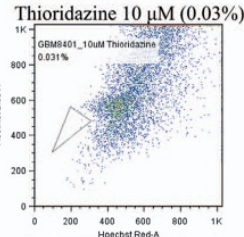

Fluphenazine $10 \mu \mathrm{M}(0.91 \%)$

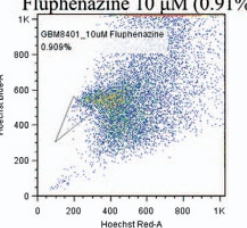

b
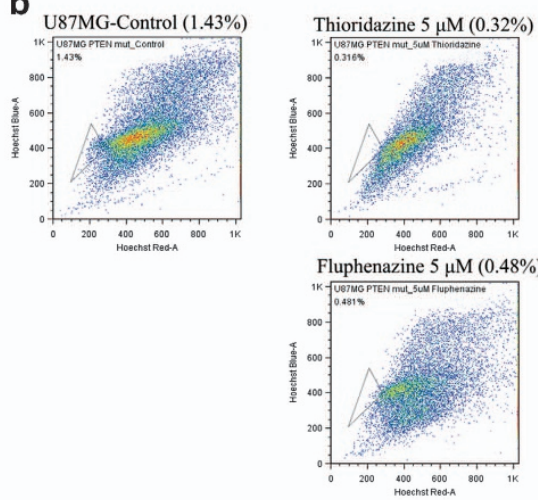

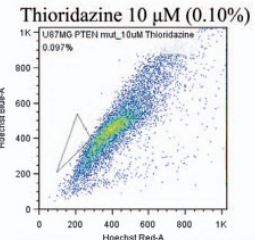

Fluphenazine $10 \mu \mathrm{M}(0.33 \%)$

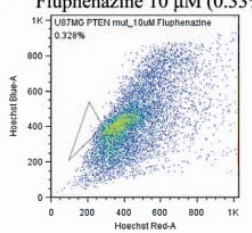

c

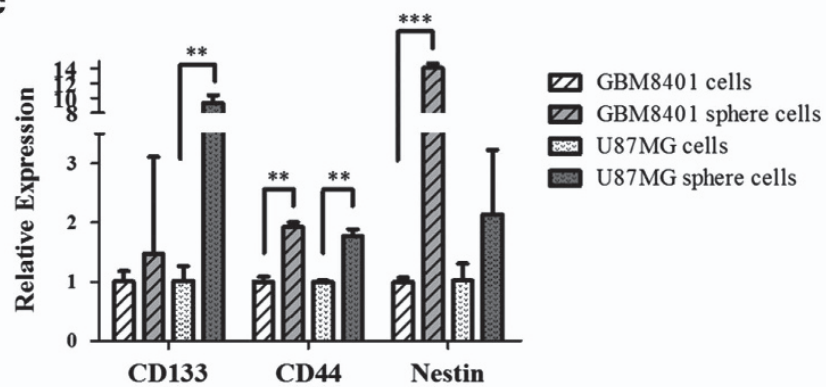

d

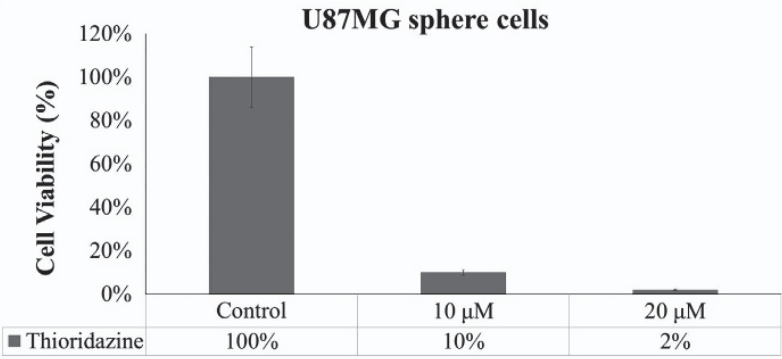

e

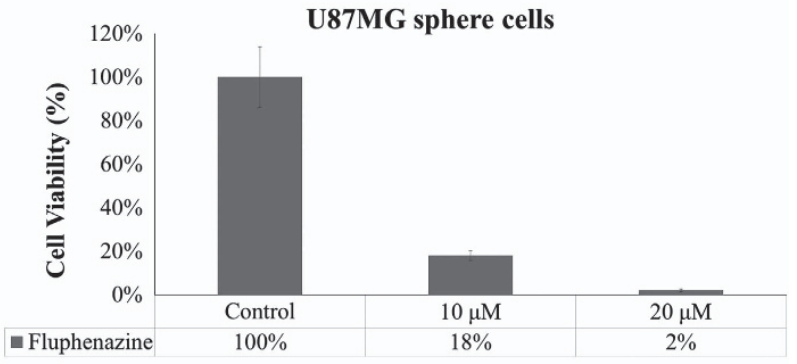

f

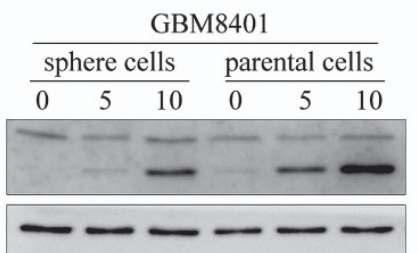

$\frac{\text { U87MG }}{\text { sphere cells } \quad \text { parental cells }}$

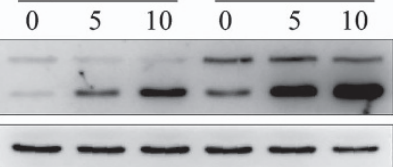

Thioridazine $(\mu \mathrm{M})$

LC3-I

LC3-II

GAPDH

Figure 4 Thioridazine and fluphenazine reduce the percentage of cancer stem-like cell side population and affect the viability of U87MG sphere cells. (a and $\mathbf{b}$ ) The cancer stem-like cells side population was significantly decreased by treatment with thioridazine (10 $\mu \mathrm{M})$ from 1.26 to $0.03 \%$ in GBM8401 cells and from 1.43 to $0.1 \%$ in U87MG cells, as determined by side population assay. (c) Quantification of GSCs gene expression with GBM8401 and U87MG sphere cells by TaqMan real-time qRT-PCR, including CD133, CD44 and Nestin. Bar graph represents the mean of triplicates \pm S.D. ${ }^{\star \star} P<0.01,{ }^{* \star \star} P<0.005$. U87MG sphere cells were treated with 10 and $20 \mu \mathrm{M}$ thioridazine (d) and fluphenazine (e) for $24 \mathrm{~h}$, respectively. The viability of the U87MG sphere cells was determined by counting cells after trypan blue staining. (f) Both sphere cells and parental cells of GBM8401 and U87MG were treated with thioridazine at 5 and $10 \mu \mathrm{M}$ for $24 \mathrm{~h}$, respectively. LC3-I and LC3-II were detected by western blotting. GAPDH was used as an internal control

Figure 3 Thioridazine modulates PI3K/Akt/p70S6K signaling pathways and induces ER stress in GBM cells. (a) GBM8401 and U87MG cells were treated with thioridazine at 5, 7.5, 10 and $15 \mu \mathrm{M}$ for $24 \mathrm{~h}$. PI3K, phospho-Akt (Ser-473), phospho-mTOR (Ser-2448) and phospho-S6K (Ser-424) and phospho-AMPK (Thr-172) were detected by western blotting. GAPDH was used as an internal control. The quantification of the western blotting intensity was normalized to internal control intensity using ImageJ. Bar graph represents the mean of triplicates \pm S.D. ${ }^{*} P<0.05,{ }^{* *} P<0.01$ compared with the control group. (b) GBM8401 and U87MG cells were treated with thioridazine at $5,7.5,10$ and $15 \mu \mathrm{M}$ for $24 \mathrm{~h}$. ER stress markers IRE1 $\alpha$, Bip and CHOP were detected by western blotting. GAPDH was used as an internal control. (c) GBM8401 cells were treated with different antipsychotic drugs at $10 \mu \mathrm{M}$ for $24 \mathrm{~h}$. DRD2, DRD1, LC3-I and LC3-II were detected by western blotting. $\alpha$-tubulin and $\beta$-actin were used as internal controls 


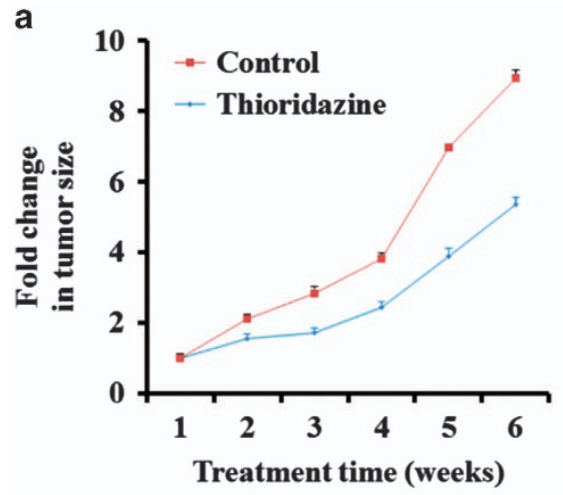

b

c

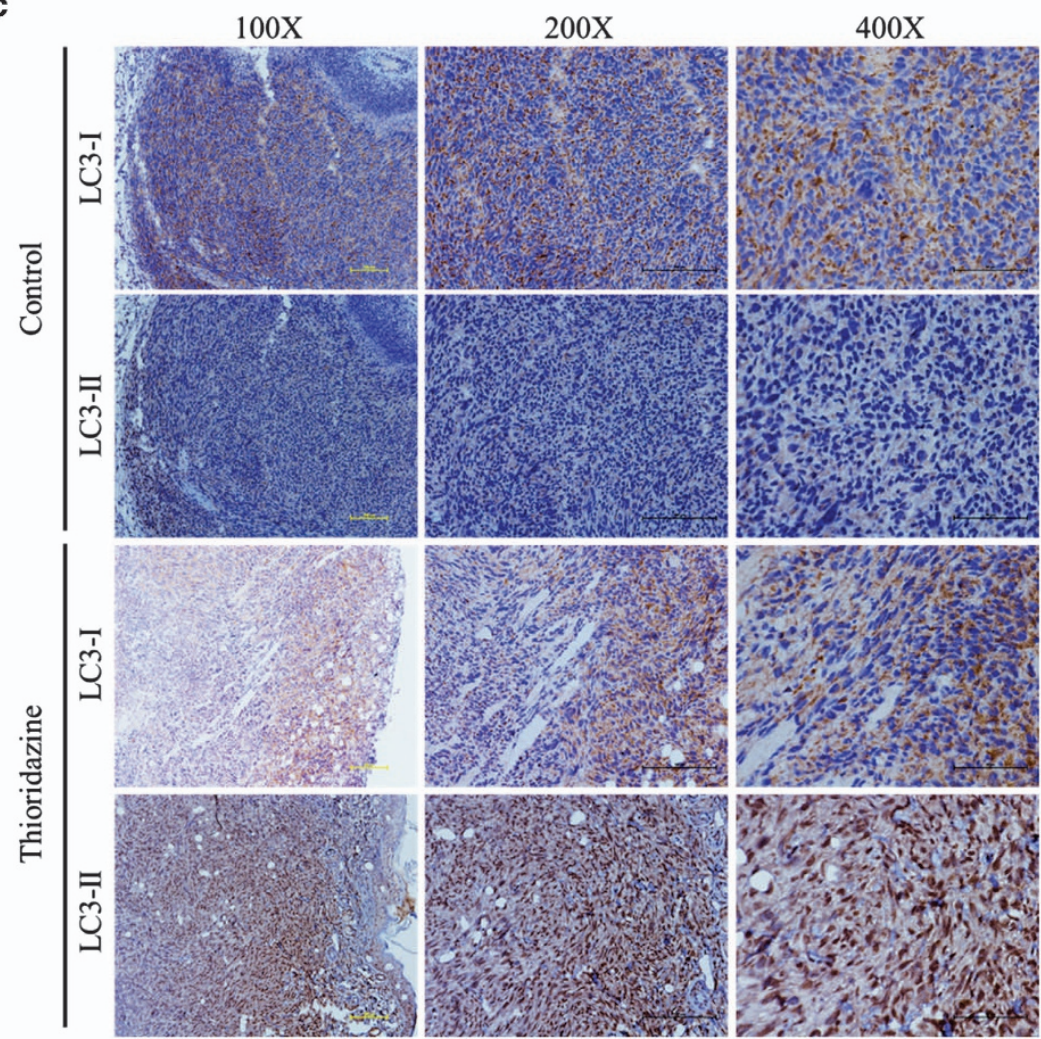

Figure 5 Thioridazine suppresses GBM tumorigenesis and induces autophagy in vivo. ( $\mathbf{a}$ and $\mathbf{b})$ U87MG cells $\left(1 \times 10^{6}\right.$ cells/injection) were subcutaneously implanted into NOD/ SCID mice and subdivided into two groups: control (DMSO) and thioridazine $(5 \mathrm{mg} / \mathrm{kg} /$ day, 5 days/week). Mice were treated by intraperitoneal injections. The tumor size was measured using caliper on a weekly basis. Mice treated with thioridazine exhibited a significantly lower tumor burden as compared with those in the control group ( $n=3$ per treatment group). (c) Representative photographs of tumor biopsies obtained from control and thioridazine-treated animals. LC3-I and LC3-II were detected by immunohistochemistry

but also provided a new strategy to search for drugs with anticancer and anticancer stem-like cell properties.

\section{Materials and Methods}

Cell lines and cell culture. Dulbecco's modified Eagle's medium (DMEM, Life Technologies, Grand Island, NY, USA; 12100-046) supplemented with 10\% fetal bovine serum (Life Technologies, 10099-133), 2 mM L-glutamine (Life Technologies, 25030-081), $100 \mathrm{U} / \mathrm{ml}$ penicillin (Life Technologies, 15140-163) and $100 \mu \mathrm{g} / \mathrm{ml}$ streptomycin (Life Technologies, 15140-163) were used to maintain human glioblastoma GBM8401 and U87MG cells (Bioresource Collection and Research Centre, BCRC, Taiwan, 60163,60360 , respectively) in a humidified incubator at $37^{\circ} \mathrm{C}$ with a atmosphere of $5 \% \mathrm{CO}_{2}$. The cells were regularly subcultured every $2-3$ days.

Chemicals. Acepromazine (A7111), Amoxapine (A129), Clozapine (C6305), Flupenthixol (F114), Fluspirilene (F100), Haloperidol (H1512), Loxapine (L106), Molindone
(M1818), Ondansetron (O3639), Pimozide (P1793), Reserpine (R0875), Spiperone (S7395), Triflupromazine (T2896), Prochlorperazine (P9178), Trifluoperazine (T8516), Thioridazine (T9025), TMZ (T2577), Chlorpromazine (C8138) and Fluphenazine (F4765) were purchased from Sigma. Perphenazine (125), Thiostrepton (522), Thioguanosine (347), Parthenolide (550) and Bepridil (368) were purchased from Prestwick. Promazine (46674) and Promethazine (46682) were purchased from Fluka.

Collection and analysis of GBM microarray data sets. Five cohorts of GBM patients (GSE4290, GSE7696, GSE14805, GSE15824 and GSE16011) 6-10 were used in this study; in total, 397 microarrays (Table 1) were obtained from the NCBI GEO website (http://www.ncbi.nlm.nih.gov/geo/). Among these five data sets, the GSE14805 data set was subjected to analysis using the Affymetrix HT-HGU133A chip, and the other data sets were analyzed using the Affymetrix HC-U133 plus 2.0 chip (Table 1). For example, the GSE4290 data set was obtained from Fine et al., ${ }^{6}$ this data set included 81 GBM samples and 23 samples from epilepsy 
patients, as nontumor controls. This data set was generated using Affymetrix HC-U133 plus 2.0 chips, which include 54675 probe sets. However, the samples used in the Cmap were profiled using Affymetrix HG-U133A gene chips, which include 22283 probe sets. Therefore, Cmap analysis was used to filter out the probe sets that do not belong to the HG-U133A platform. To analyze the GBM microarrays, the raw fluorescence intensity data within the raw CEL files of these data sets were preprocessed and normalized using the Bioconductor Affy package, based on the Robust Multichip Average algorithm with $\mathrm{R}$ language from Bioconductor (http://www.bioconductor.org). The logarithm to the base 2 of the intensity in the tumor and the control sample was used as the expression value for each probe.

In silico drug screening via the Cmap. To identify significant differentially expressed genes from microarrays with GBM and normal brain samples, we used the two sample $t$-test $(P$-value $<0.0001)$ with at least fourfold change cutoff. For each GBM sample and nontumor sample, 22283 means of expression (one for each probe set) were calculated. The probe sets with significant mean ratios (greater than a fourfold change) were selected as the genes that were differentially expressed. The details of the significant probe sets are summarized in Table 1. These probe sets were used to query up to 7000 expression profiles stored in the Cmap database. For example, to identify the differentially expressed probe sets from the GSE4290 data set using the volcano plot method, 120 upregulated and 286 downregulated probe sets were selected and used to query the Cmap. To identify potential GBM treatments, only drugs with a $P$-value of less than 0.05 and a negative enrichment score were retained. The details of the potential drugs from the five GBM data sets are shown in Supplementary Information, Supplementary Table S1.

Cell viability assay. The viability of cell was measured using the MTT assay. Briefly, cells (1500 cells/well) in 96-well plates were treated with various concentrations of the tested agents for various time points. After treatment, $10 \%$ stock MTT reagent $(0.02 \mathrm{mg}$ MTT from Sigma in $10 \mathrm{ml}$ phosphate-buffered saline (PBS) for stock reagent, M5655) was added to each well. After another $3-5 \mathrm{~h}$, the cells were dissolved in DMSO for $10 \mathrm{~min}$ at room temperature. The absorbance of each well at $570 \mathrm{~nm}$ was measured using a microplate reader.

Clonogenic assay. GBM8401 cells were seeded at a density of $1 \times 10^{3}$ cells per well in six-well plates. Each well was given $2 \mathrm{ml} \mathrm{DMEM} \mathrm{medium} \mathrm{to} \mathrm{culture} \mathrm{the}$ GBM8401 cells. After $24 \mathrm{~h}$, thioridazine, fluphenazine and other drugs were added, and cells were treated for 14 days. The culture medium was changed on days 4,8 and 12, and fresh drugs were added at this time. After treatment, cells were washed carefully with PBS. The colonies were then incubated with fix solution (acetic acid: methanol $=1: 3$ ). After being stained with $0.5 \%$ crystal violet in methanol, the cells were washed with tap water. The colonies were counted manually.

Microarray analysis. Human GBM cell line, GBM8401 cells, were treated with $10 \mu \mathrm{M}$ thioridazine for $6 \mathrm{~h}$. After treatments, total RNA was isolated using the RNeasy Mini kit (QIAGEN, Valencia, CA, USA; 74106). Gene expression was analyzed using the Affymetrix U133plus 2 array. The quality of the total RNA for microarray analysis was determined using UV spectrophotometer and Agilent 2100 Bioanalyzer and had an OD260/OD280 ratio ranging from 1.9 to 2.1. Total RNA was used to synthesize CDNA and was then converted to CRNA. CRNA was labeled, fragmented and quality-checked using Bioanalyzer. According to instructions provided by Affymetrix (http://www.affymetrix.com/support/technical/manuals.affx), hybridization, washing and staining procedures were performed. The images transformed into the text files contain the intensity information using the GeneChip Operating Software, developed by Affymetrix. The microarray data sets were determined using the GeneSpring 7.31 software (QIAGEN, Agilent, Santa Clara, CA, USA).

Consensus PathDB data analysis. To analyze the thioridazine-elicited effect on GBM8401 cells, we first selected the differentially expressed genes, which were defined as fold changes greater than 1.75 with the greatest difference in expression levels, from the Affymetrix HG-U133_Plus_2 microarray profiling, and these genes were then subjected to ConsensusPathDB (http://cpdb.molgen.mpg.de/) analysis (Supplementary Information, Supplementary Table S2). The second set of differentially expressed genes subjected to the ConsensusPathDB analysis was obtained from the original Cmap data sets, which included 14 thioridazine-treated
Affymetrix U133A arrays. Genes were selected based on at least a twofold change and appearance in at least 3 of the 14 arrays.

Microwestern. GBM8401 cells were treated with thioridazine at concentrations ranging from 5 to $15 \mu \mathrm{M}$, or fluphenazine 10 and $20 \mu \mathrm{M}$ for $24 \mathrm{~h}$, respectively. At the end of treatment, cells were lysed with lysis buffer ( $240 \mathrm{mM}$ Tris-acetate, $1 \%$ sodium dodecyl sulfate (SDS), $0.5 \%$ glycerol and $5 \mathrm{mM}$ EDTA), and protein expression was examined via microwestern (Supplementary Information, Supplementary Figure S1) as previously described. ${ }^{17}$ The position of primary antibodies is summarized in Supplementary Information, Supplementary Table S3. Primary antibodies, including stress-activated kinases (SAPK)/c-Jun N-terminal kinases (JNK; 9258), Src (2109), bcl-2-associated X protein (2772), c-Myc (5605), c-Raf (9422), glycogen synthase kinase 3 beta (9315), heat shock protein 90 (4874), janus kinase 2 (3230), p38 $\alpha$ (2371), p70S6K (2708), phospho-AMPK (Thr-172; 4188), phospho-p38 (Thr-180/ Tyr-182; 4511S), phospho-PDGF $\beta$ (Tyr-1009) (3124S), phospho-Raptor (Ser-792; 2083), phospho- retinoblastoma protein (Ser-780; 3590S), phospho-SAPK/JNK (Thr-183/Tyr-185; 4668S), phospho-Tuberin/ tuberous sclerosis 2 (Thr-1462; 3617) and phospho-VEGF Receptor 2 (Tyr-1059; 3817S), were obtained from Cell Signaling (Danvers, MA, USA). All other antibodies were obtained from Millipore (Bedford, MA, USA).

Western blotting analysis. Cells were lysed with lysis buffer (Thermo, Waltham, MA, USA; 89900) containing $25 \mathrm{mM}$ Tris- $\mathrm{HCl}(\mathrm{pH} 7.6), 150 \mathrm{mM} \mathrm{NaCl}, 1 \%$ NP- $40,1 \%$ sodium deoxycholate and $0.1 \%$ SDS. Total protein was isolated and subjected to SDS-polyacrylamide gel electrophoresis and electrotransferred on polyvinylidene difluoride membranes (Millipore, IPVH00010). The primary antibodies, including Bip (1: 1000; 3177), CHOP (1: 1000; 2895), IRE1 $\alpha$ (1: 1000; 3294), phospho-mTOR (Ser-2448; 1 : 1000; 5536S), mTOR (1 : 1000; 2983S), PI3K (p110 $\alpha ; 1: 1000 ; 4249 S)$, phospho-Akt (Ser-473; $1: 1000 ; 4060 S)$, Akt (1:1000; 2938S), phospho-p70S6K (Ser-424; 1: 1000; 9205S), p70S6K (1: 1000; 2708S), phospho-AMPK (Thr-172; 1:1000; 2535S), AMPK (1:1000; 2532S), c-PARP (1:1000; 5625P), c-Caspase-3 (1:1000; 9664S) and Caspase-3 (1:1000; 9662S), p-Raptor (Ser-792; 1:1000; 2083S), Beclin1 (1:1000; 3495S) and c-Caspase-8 $(1: 1000 ; 8592)$ were obtained from Cell Signaling. DRD2 $(1: 1000 ;$ ab88074) and Dopamine receptor D1 (DRD1, $1: 1000$; ab40653) and $\beta$-actin (1:1000; ab3280) were from Abcam (Cambridge Science Park, UK), and LC3 was from Abgent (San Diego, CA, USA; $1: 1000 ;$ AP1802a), and GAPDH (1:10000; GTX100283) and $\alpha$-tubulin $(1: 5,000$; GTX112141) were from GeneTex (Irvine, CA, USA). The secondary antibodies for anti-rabbit and anti-mouse horseradish preoxidase (HRP) conjugation were from Chemicon (Shinagawa-ku, Tokyo, Japan; 12-348 and 12-349, respectively). The protein detection was performed with enhanced chemiluminescence (GE Healthcare, Pittsburgh, PA, USA; RPN2108) method captured by a Luminescence Imaging System (LAS-4000, Fuji Photo Film Co, Ltd, Minato-ku, Tokyo, Japan). After scanning western blots into a computer, individual bands were analyzed for optical density using ImageJ.

Determination of caspase 8 activity. U87MG and GBM8401 cells seeded at a density of $2.5 \times 10^{4}$ cells per well in 96-well plates were treated with 5-15 $\mu \mathrm{M}$ thioridazine. After $24 \mathrm{~h}$, the substrate of caspase 8 was added to the samples and incubated for $30 \mathrm{~min}$. The activity was detected using luminescence.

Detection of AVOs with AO staining using flow cytometry. $\mathrm{AO}$ is a fluorescent weak base that accumulates in acidic spaces and fluoresces bright red. ${ }^{36}$ AVOs, representing formation of autophagosomes and autolysosomes, were determined using flow cytometry after cells were stained with $\mathrm{AO}$. After drug treatments, cells were stained with AO $(1 \mu \mathrm{g} / \mathrm{ml}$, Sigma, St. Louis, MO, USA; A6014) for $20 \mathrm{~min}$ at room temperature, washed twice with PBS, removed from the dish with trypsin-EDTA (Life Technologies, R-001-100) and collected in phenol redfree growth medium. AVOs were measured using a BD FACSCanto, followed by analysis with the FACS Diva software (BD Bioscience, San Jose, CA, USA).

Immunofluorescence staining. U87MG cells on the coverslips in 12-well plate were pretreated in the presence and absence of $50 \mathrm{nM}$ Bafilomycin A1 (Sigma, B1793) for $4 \mathrm{~h}$ before $7.5 \mu \mathrm{M}$ thioridazine treatment for $24 \mathrm{~h}$. After being fixed with $3.7 \%$ formaldehyde in Hank's balanced salt solution (HBSS) at room temperature, the cells were blocked with $5 \%$ bovine serum albumin (Millipore, 810037) in HBSS. Followed by incubated with anti-LC3 antibody (rabbit polyclonal lgG; 1: 100; Abgent, AP1802a) and LAMP-2 (mouse monoclonal IgG; 1:100; Abcam, ab25631), the primary antibodies were examined using Alexa Fluor 
488-conjugated anti-rabbit antibody (1: 1000; Life Technologies, A11008) and Alexa Fluor 568-conjugated anti-mouse antibody (1:1000; Life Technologies, A11004). DAPI ( $2 \mu \mathrm{g} / \mathrm{ml}$, Sigma, D9542) was used to stain the nuclei after being mounted with ProLong Gold antifade reagents (Life Technologies, P36930). The images were obtained using an Olympus BX61 confocal microscope using $\times 60$ objective.

Identification of side population by using flow cytometry. U87MG and GBM8401 cells were treated with thioridazine at 5 and $10 \mu \mathrm{M}$ or fluphenazine at 5 and $10 \mu \mathrm{M}$ for $24 \mathrm{~h}$, respectively. Cells were detached by trypsinization (Life Technologies, 12604013), following by resuspension at $1 \times 10^{6} \mathrm{cell} / \mathrm{s} / \mathrm{ml}$ in $3 \%$ fetal calf serum and $10 \mathrm{mM}$ HEPES-supplemented HBSS (Life Technologies, 15630-080). Cells were then incubated with Hoechst 33342 (Sigma, 14533) at $37^{\circ} \mathrm{C}$ for $90 \mathrm{~min}$, alone or with $50 \mu \mathrm{M}$ verapamil (Sigma, V4629), for inhibition of the verapamil-responsive $A B C$ transporter. Cells were then centrifuged at $300 \times g$ and resuspended in ice-cold HBSS. Cells were placed on ice to inhibit efflux of the Hoechst dye, followed by addition of $1 \mu \mathrm{g} / \mathrm{ml}$ propidium iodide (BD Bioscience, 556463 ) to distinguish dead cells. Finally, a single-cell suspension was generated by filtering the cells through a $40-\mu \mathrm{m}$ cell strainer (BD Bioscience) to obtain. Dualwavelength analysis and purification were then performed using a dual-laser FACS Vantage SE machine (BD Bioscience). We excited Hoechst 33342 with a $355-n m$ UV light, followed by emission of blue fluorescence with a 450/20 band-pass filter as well as red fluorescence with a $675-\mathrm{nm}$ edge filter long-pass. A 610-nm beamsplitter or so-called 'dichroic mirror shortpass' was used to separate the emitted light per wavelength.

Real-time quantitative RT-PCR. Total RNA was extracted from U87MG cells and sphere cells using the NucleoSpin RNA II kit (MACHEREY-NAGEL, Bethlehem, PA, USA) according to the manufacturer's protocol. For CDNA synthesis, RNA was reverse-transcribed into cDNA using ThermoScript RT-PCR System (Invitrogen, Grand Island, NY, USA). Gene expression was quantified by real-time quantitative RT-PCR using TaqMan probe (Life Technologies). The relative quantities of gene mRNA against an internal control, GAPDH, were detected by following a $\Delta C_{t}$ method. The difference $\left(\Delta C_{t}\right)$ between the mean values in the duplicated samples of target gene and those of GAPDH were calculated by Microsoft Excel and the relative quantified value was expressed as $2^{-\Delta C}$.

Tumor spheroid assay. To evaluate the formation of tumor spheroids, we cultured GBM8401 and U87MG cells in HEScGRO serum-free medium (Chemicon SCM020) supplemented with NeuroCult NS-A (STEMCELL Technologies, Vancouver, BC, Canada; 5751), $20 \mathrm{ng} / \mathrm{ml}$ human epidermal growth factor and $10 \mathrm{ng} / \mathrm{ml} \mathrm{hFGF}$. Cells were seeded at $1 \times 10^{3} \mathrm{cell} / \mathrm{s} / \mathrm{ml}$ in 12-well, low-adhesion plates. The generated spheroids (tight, spherical, non-adherent cell-masses $>90 \mu \mathrm{m}$ in diameter) were counted, followed by measurement of at least 50 spheroids per group using an ocular micrometer. For the secondary spheroid formation assay, we mechanically dissociated the primary spheroids and processed exactly as for the primary assay. To estimate the percentage of spheroid-forming cells, we seeded one cell per well in 96-well plates.

Xenograft experiments. U87MG cells $\left(1 \times 10^{6}\right.$ cells/injection) were subcutaneously implanted into the flank of NOD/SCID mice. The mice were randomly distributed into two groups (three mice/group): control (DMSO as the vehicle) and thioridazine ( $5 \mathrm{mg} / \mathrm{kg} /$ day, 5 days/week). The animal study was performed under the strict adherence to the Labortory Animal Use Protocol by Taipei Medical Univeristy (Protocol Approval number: LAC-2013-0086). The treatment began 1 week posttumor implantation. The tumor volume was then measured using caliper on a weekly basis. Tumor volume was measured using a conventional formula: volume $(V)=\left(W^{2} \times L\right) / 2$ where $W=$ width and $L=$ length. The changes in tumor volume (fold change) was then plotted against time. Representative photographs of tumor biopsies obtained from control and thioridazine-treated animals.

Histology and immunohistochemical staining. Tumor tissues were collected from xenografted mice, and then fixed with formalin and embedded in paraffin. After antigen retrieval of deparaffinized slides, they were probed with antiLC3-I antibody (1:100; Cell Signaling, 4599P) or anti-LC3-II antibody (1: 100; Cell Signaling, 3868P). Later slides were washed and incubated in a biotinylated link universal antiserum, and then in HRP-conjugated streptavidin (LSAB one kit, GeneCopoeia, Rockville, MD, USA; VB-6015). Slides were rinsed and colordeveloped with the chromogen, 3, 3-diaminobenzidine hydrochlorides. Lastly, tissue sections were washed with distilled water, counterstained with Mayer's hematoxylin and mounted with DPX mountant. Pictures were then taken with a Photometrics CoolSnap CF color camera (Nikon, Lewisville, TX, USA).

\section{Conflict of Interest}

The authors declare no conflict of interest.

Acknowledgements. This research was supported by the grant from the Ministry of Education, Taiwan (Aim for the Top University Plan, 103AC-T503 to C-YFH), the Center of Excellence for Cancer Research at Taipei Veterans General Hospital, Taiwan (Health and Welfare Surcharge of Tobacco Products, MOHW103TD-B-111-02 to C-YFH), the National Science Council, Taiwan (NSC 101-2313B-003-002-MY3 to C-LS and NSC 102-2314-B-038-022-MY2 to JW-P) and the Ministry of Science and Technology (MOST 103-2325-B-010-006 to C-YFH).

1. Kaza N, Kohli L, Roth KA. Autophagy in brain tumors: a new target for therapeutic intervention. Brain Pathol 2012; 22: 89-98.

2. Mason WP, Cairncross JG. Drug Insight: temozolomide as a treatment for malignant gliomaimpact of a recent trial. Nat Clin Pract Neurol 2005; 1: 88-95.

3. Stupp R, Mason WP, van den Bent MJ, Weller M, Fisher B, Taphoorn MJ et al. Radiotherapy plus concomitant and adjuvant temozolomide for glioblastoma. N Engl J Med 2005; 352: 987-996.

4. Chou FH, Tsai KY, Su CY, Lee CC. The incidence and relative risk factors for developing cancer among patients with schizophrenia: a nine-year follow-up study. Schizophr Res 2011; 129: $97-103$.

5. Tabares-Seisdedos R, Rubenstein JL. Inverse cancer comorbidity: a serendipitous opportunity to gain insight into CNS disorders. Nat Rev 2013; 14: 293-304.

6. Sun L, Hui AM, Su Q, Vortmeyer A, Kotliarov Y, Pastorino S et al. Neuronal and gliomaderived stem cell factor induces angiogenesis within the brain. Cancer Cell 2006; 9: 287-300.

7. Murat A, Migliavacca E, Gorlia T, Lambiv WL, Shay T, Hamou MF et al. Stem cell-related 'self-renewal' signature and high epidermal growth factor receptor expression associated with resistance to concomitant chemoradiotherapy in glioblastoma. J Clin Oncol 2008; 26: 3015-3024.

8. Hodgson JG, Yeh RF, Ray A, Wang NJ, Smirnov I, Yu M et al. Comparative analyses of gene copy number and mRNA expression in glioblastoma multiforme tumors and xenografts. Neuro Oncol 2009; 11: 477-487.

9. Grzmil M, Morin P Jr., Lino MM, Merlo A, Frank S, Wang Y et al. MAP kinase-interacting kinase 1 regulates SMAD2-dependent TGF-beta signaling pathway in human glioblastoma. Cancer Res 2011; 71: 2392-2402.

10. Gravendeel LA, Kouwenhoven MC, Gevaert O, de Rooi JJ, Stubbs AP, Duijm JE et al. Intrinsic gene expression profiles of gliomas are a better predictor of survival than histology. Cancer Res 2009; 69: 9065-9072.

11. Shats I, Gatza ML, Chang JT, Mori S, Wang J, Rich J et al. Using a stem cell-based signature to guide therapeutic selection in cancer. Cancer Res 2011; 71: 1772-1780.

12. Suva ML, Riggi N, Janiszewska M, Radovanovic I, Provero $P$, Stehle JC et al. EZH2 is essential for glioblastoma cancer stem cell maintenance. Cancer Res 2009; 69: 9211-9218.

13. Wong DJ, Liu H, Ridky TW, Cassarino D, Segal E, Chang HY. Module map of stem cell genes guides creation of epithelial cancer stem cells. Cell Stem Cell 2008; 2: 333-344.

14. Krakstad C, Chekenya M. Survival signalling and apoptosis resistance in glioblastomas: opportunities for targeted therapeutics. Mol Cancer 2010; 9: 135.

15. Wick W, Weller M, Weiler M, Batchelor T, Yung AW, Platten M. Pathway inhibition: emerging molecular targets for treating glioblastoma. Neuro Oncol 2011; 13: 566-579.

16. Lino MM, Merlo A. PI3Kinase signaling in glioblastoma. J Neurooncol 2011; 103 417-427.

17. Ciaccio MF, Wagner JP, Chuu CP, Lauffenburger DA, Jones RB. Systems analysis of EGF receptor signaling dynamics with microwestern arrays. Nat Methods 2010; 7 : 148-155.

18. Takeuchi H, Kondo $\mathrm{Y}$, Fujiwara K, Kanzawa $\mathrm{T}$, Aoki $\mathrm{H}$, Mills $\mathrm{GB}$ et al. Synergistic augmentation of rapamycin-induced autophagy in malignant glioma cells by phosphatidylinositol 3-kinase/protein kinase B inhibitors. Cancer Res 2005; 65: 3336-3346.

19. Filippi-Chiela EC, Villodre ES, Zamin LL, Lenz G. Autophagy interplay with apoptosis and cell cycle regulation in the growth inhibiting effect of resveratrol in glioma cells. PLOS ONE 2011; 6: e20849.

20. Fu J, Shao CJ, Chen FR, Ng HK, Chen ZP. Autophagy induced by valproic acid is associated with oxidative stress in glioma cell lines. Neuro Oncol 2010; 12: 328-340.

21. Song X, Kim SY, Zhang L, Tang D, Bartlett DL, Kwon YT et al. Role of AMP-activated protein kinase in cross-talk between apoptosis and autophagy in human colon cancer. Cell Death Dis 2014; 5: e1504.

22. Roach PJ. AMPK -> ULK1 -> autophagy. Mol Cell Biol 2011; 31: 3082-3084.

23. Tuynder M, Fiucci G, Prieur S, Lespagnol A, Géant A, Beaucourt S et al. Translationally controlled tumor protein is a target of tumor reversion. Proc Natl Acad Sci USA 2004; 101 : 15364-15369. 
24. Chen K, Huang C, Yuan J, Cheng H, Zhou R. Long-term artificial selection reveals a role of TCTP in autophagy in mammalian cells. Mol Biol Evol 2014; 31: 2194-2211.

25. Rinnerthaler M, Lejskova R, Grousl T, Stradalova V, Heeren G, Richter K et al. Mmi1, the yeast homologue of mammalian TCTP, associates with stress granules in heat-shocked cells and modulates proteasome activity. PLOS ONE 2013; 8: e77791.

26. Zungu M, Schisler JC, Essop MF, McCudden C, Patterson C, Willis MS. Regulation of AMPK by the ubiquitin proteasome system. Am J Pathol 2011; 178: 4-11.

27. Emdad L, Qadeer ZA, Bederson LB, Kothari HP, Uzzaman M, Germano IM. Is there a common upstream link for autophagic and apoptotic cell death in human high-grade gliomas? Neuro Oncol 2011; 13: 725-735.

28. Li J, Zhu S, Kozono D, Ng K, Futalan D, Shen $Y$ et al. Genome-wide shRNA screen revealed integrated mitogenic signaling between dopamine receptor D2 (DRD2) and epidermal growth factor receptor (EGFR) in glioblastoma. Oncotarget 2014; 5 : 882-893.

29. Burstein ES, Ma J, Wong S, Gao Y, Pham E, Knapp AE et al. Intrinsic efficacy of antipsychotics at human D2, D3, and D4 dopamine receptors: identification of the clozapine metabolite N-desmethylclozapine as a D2/D3 partial agonist. J Pharmacol Exp Ther 2005; 315: $1278-1287$.

30. Weller M, Stupp R, Reifenberger G, Brandes AA, van den Bent MJ, Wick W et al. MGMT promoter methylation in malignant gliomas: ready for personalized medicine? Nat Rev Neurol 2010; 6: 39-51.

31. Riddick G, Fine HA. Integration and analysis of genome-scale data from gliomas. Nat Rev Neurol 2011; 7: 439-450.

32. Parsons DW, Jones S, Zhang X, Lin JC, Leary RJ, Angenendt P et al. An integrated genomic analysis of human glioblastoma multiforme. Science 2008; 321: 1807-1812.
33. Singh SK, Clarke ID, Terasaki M, Bonn VE, Hawkins C, Squire J et al. Identification of a cancer stem cell in human brain tumors. Cancer Res 2003; 63: 5821-5828.

34. Sachlos E, Risueno RM, Laronde S, Shapovalova Z, Lee JH, Russell J et al. Identification of drugs including a dopamine receptor antagonist that selectively target cancer stem cells. Cell 2012; 149: 1284-1297

35. Gil-Ad I, Shtaif B, Levkovitz Y, Dayag M, Zeldich E, Weizman A et al. Characterization of phenothiazine-induced apoptosis in neuroblastoma and glioma cell lines: clinical relevance and possible application for brain-derived tumors. J Mol Neurosci 2004; 22: 189-198.

36. Traganos F, Darzynkiewicz Z. Lysosomal proton pump activity: supravital cell staining with acridine orange differentiates leukocyte subpopulations. Methods Cell Biol 1994; 41: 185-194.

Cell Death and Disease is an open-access journal published by Nature Publishing Group. This work is licensed under a Creative Commons Attribution 4.0 International License. The images or other third party material in this article are included in the article's Creative Commons license, unless indicated otherwise in the credit line; if the material is not included under the Creative Commons license, users will need to obtain permission from the license holder to reproduce the material. To view a copy of this license, visit http://creativecommons.org/licenses/by/4.0/

Supplementary Information accompanies this paper on Cell Death and Disease website (http://www.nature.com/cddis) 